\title{
Cartilage markers in synovial fluid in symptomatic knee osteoarthritis
}

\author{
Ingemar F Petersson, Lars Sandqvist, Björn Svensson, Tore Saxne
}

\begin{abstract}
Objective-To investigate if the relative content of aggrecan and cartilage oligomeric matrix protein (COMP) in synovial fluid lavage samples differs between individuals with knee pain with or without evidence of radiological knee osteoarthritis (joint space narrowing). Methods-In a community based cohort of 204 individuals aged 35-54 years with chronic (>3 months duration) knee pain, a subgroup of 45 subjects with radiographic osteoarthritis, grade I according to the Ahlbäck classification, was randomly chosen; 45 individuals, age and sex matched from the same cohort with chronic knee pain but with normal radiographs, served as controls. Knee joint fluid was obtained by a standardised lavage procedure. The concentrations of aggrecan core protein epitopes and a cartilage matrix protein (COMP) were determined by immunoassays and the aggrecan/COMP concentration ratio was calculated.

Results-The aggrecan/COMP ratio was higher $(P<0.001)$ in the group with radiographic osteoarthritis than in the control group.

Conclusions-The higher aggrecan/COMP ratios in osteoarthritis could reflect increased cartilage matrix turnover in osteoarthritis with predominant release of aggrecan fragments. Synovial fluid analysis of cartilage markers holds promise as a useful means of monitoring changes in the cartilage turnover in studies of pathogenic mechanisms in osteoarthritis.

(Ann Rheum Dis 1997;56:64-67)
\end{abstract}

Spenshult's Hospital

for Rheumatic

Diseases, Oskarström,

Sweden

I F Petersson

L Sandqvist

B Svensson

Correspondence to:

Dr Ingemar Petersson,

Spenshult's Hospital for

Rheumatic Diseases, S-313

92 Oskarström, Sweden.

E-mail:

ingemar.petersson@spenshult.se

Accepted for publication

4 September 1996
However, radiographic changes correspond to advanced tissue alterations following a sequence of events at the molecular level. ${ }^{34}$
Osteoarthritis is a common disease causing significant disability and discomfort. ${ }^{2}$ Clinic patients with advanced often end sta diagnosing less severe cases. The standard diagnostic technique for knee osteoarthritis is evaluation of plain weight bearing radiographs.
Osteoarthritis leads in many cases to destruction of one or more joints. There is, however, no known treatment that modifies the progression of the disease. A prerequisite for developing disease modifying agents is a reliable method for studying early pathogenic mechanisms. An ongoing development of technology for identifying processes in cartilage by measurements of cartilage macromolecules in synovial fluid offers promise. Information on differences between different types of arthritis has been documented. ${ }^{5}$ Aggrecan molecules in intact or fragmented form may be released into synovial fluid as a result of increased biosynthesis as well as cartilage degradation. Thus increased concentrations of aggrecan fragments have been found in experimental models $^{6}$ as well as in several arthritic conditions including rheumatoid arthritis and osteoarthritis. $^{7}$ Of particular interest are the high concentrations in synovial fluid from patients with knee trauma in whom high levels may persist for several years, perhaps as a sign of early osteoarthritis. ${ }^{8}$ More recently, an assay for an additional cartilage matrix protein COMP (cartilage oligomeric matrix protein), released into the synovial fluid in joint disease has been described. ${ }^{9}$ Raised synovial fluid concentrations of COMP have been found in rheumatoid arthritis and in osteoarthritis. ${ }^{9}$ The release of this marker seems to be increased in joint trauma and in early stage osteoarthritis. ${ }^{10}$ Although the synovial fluid concentrations of both macromolecules were increased in the osteoarthritis patients in these hospital based studies, the relative release of aggrecan seemed higher than the release of COMP, as determined by the synovial fluid ratio of the markers. ${ }^{910}$ In one of these studies we found higher relative content of COMP in synovial fluids of healthy athletes. ${ }^{10}$ These observations suggested different patterns of involvement of these cartilage components in osteoarthritis as well as different release patterns of these components between normal and osteoarthritic cartilage. To verify this hypothesis we investigated the relative content of aggrecan and COMP in synovial fluid lavage samples of two matched groups of individuals with knee pain recruited from the general population. One of the groups had knee pain with radiographic evidence of knee osteoarthritis (joint space narrowing in the tibiofemoral joint). The other 
was a matched group of individuals with knee pain but without such radiographic changes. For comparison samples were also obtained from a small group of healthy non-athletic hospital workers.

The lavage approach was chosen since very few individuals were expected to have synovial fluid in amounts possible to aspirate without a lavage procedure. A future aim is to elucidate whether measurements of these markers can be used to detect biochemical changes in osteoarthritis before radiographic changes appear.

\section{Methods}

STUDY DESIGN

The study cohort was recruited by a questionnaire with regard to chronic knee pain $(>3$ months during the last 12 months). The target was a population $(n=2000)$ in a district in southern Sweden in the age group 35-54 years. Two hundred and seventy nine out of 2000 individuals had chronic knee pain, and 204 accepted clinical and radiographic examination. The radiographs were performed under weight bearing conditions and were classified according to the Ahlbäck classification for the tibiofemoral joint. ${ }^{11}$ Sixty six of these 204 individuals had radiographic osteoarthritis of the tibiofemoral joint. The majority of these subjects (56/66) had Ahlbäck grade I changes (joint space narrowing) corresponding to Kellgren \& Lawrence grade $3 .{ }^{12}$ A subset of 45 individuals with Ahlbäck grade I changes in whom a sufficient amount of knee joint lavage fluid (see below) was available was selected for this study. Forty five other subjects from the cohort (matched for sex, age, and body mass index (BMI)) with chronic knee pain but with normal radiographs, formed the primary control group (table 1). A second control group consisted of eight healthy individuals (hospital workers) with no history of knee symptoms.

ASPIRATION OF KNEE JOINT FLUID

The procedure described by Geborek et al ${ }^{13}$ was used after approval from the ethics committee and after permission from each individual to obtain knee joint fluid. Arthrocentesis of the supralateral pouch was performed with a Teflon cannula (inner diam-

Table 1 Characteristics of the three groups studied. Figures denote median (range)

\begin{tabular}{llll}
\hline & $\begin{array}{l}\text { OA grade I (joint space } \\
\text { narrowing) }\end{array}$ & Controls with knee pain & Healthy controls \\
\hline No & 45 & 45 & 8 \\
Age (years) & $46.2(35-54)$ & $45.3(35-54)$ & $38(29-55)$ \\
Sex (male/female) & $24 / 21$ & $25 / 20$ & $8 / 0$ \\
BMI & 26.1 & 25.1 & - \\
\hline
\end{tabular}

BMI, body mass index; OA, osteoarthritis. eter $1.4 \mathrm{~mm}$ ) using the lateral approach after local anaesthesia subcutaneously. After aspiration to dryness and removal (if there was any excess synovial fluid), $20 \mathrm{ml}$ of physiological saline was injected. A mixing procedure including knee bendings and external alternating pressure on the patella (10 of each during 1-2 min) was performed to ensure sufficient mixing. The knee joint was then aspirated to dryness. Aspiration of fluid from the healthy individuals was performed without a lavage procedure. The fluid was centrifuged at $2150 \mathrm{~g}$ for $20 \mathrm{~min}$ and the supernatant stored at $-80^{\circ} \mathrm{C}$ until analysis.

ASSAY OF AGGRECAN AND COMP

Aggrecan and COMP were quantified either in undiluted (healthy volunteers, $\mathrm{n}=8$ ) or in diluted lavage fluid (individuals with chronic knee pain, $n=90$ ) by specific enzyme linked immunoassays as previously described. ${ }^{59}$ The polyclonal antiserum for aggrecan recognises core protein epitopes, preferentially in the chondroitin-sulphate-rich region

STATISTICAL METHODS

The Mann-Whitney test was used for comparison between the groups. A P value $<0.05$ was considered significant.

\section{Results}

The ratio between the aggrecan and COMP concentrations in the lavage fluid was significantly higher in the group with radiographic osteoarthritis grade I compared to the ratios in the group with knee pain but without radiographic changes (table 2, figure), $\mathrm{P}<0.001$. In fact, 17 subjects in the osteoarthritis group had ratios above 1 compared to only 2 in the control group with knee pain (figure). Consequently, 28 cases in the osteoarthritis group and 43 cases in the group with normal radiographs had ratios equal to or below 1 , that is, there was a considerable overlap between the groups. The aggrecan /COMP ratios in the healthy control group did not differ from the ratios in the symptomatic group without radiographic changes (table 2, figure).

Since undiluted joint fluid was available only in a few cases and the degree of dilution during the lavage procedure could not be determined, a direct comparison between concentrations in the two groups was not feasible. However, in all cases synovial fluid concentrations of both aggrecan and COMP were readily measurable within the detection limits of the assays despite sample dilution which is shown in table 2 .

Table 2 Concentrations $\left(\mu \mathrm{g} \mathrm{ml} \mathrm{l}^{-1}\right.$ ) and ratios in synovial fluid (diluted or undiluted) or aggrecan and COMP in the different groups

\begin{tabular}{|c|c|c|c|}
\hline & Median aggrecan concentration (range) & Median COMP concentration (range) & Ratio aggrecan/COMP concentrations \\
\hline $\begin{array}{l}\text { Chronic knee pain with } \mathrm{OA}(\mathrm{n}=45 \text {, } \\
\text { diluted fluid) }\end{array}$ & $6.9(2.0-34.4)$ & $7.6(1.8-118.6)$ & $0.8(0.1-2.5)$ \\
\hline $\begin{array}{l}\text { Chronic knee pain without } \mathrm{OA} \text { ( } \mathrm{n}=45 \text {, } \\
\text { diluted fluid) }\end{array}$ & $4.8(2.0-47.6)$ & $7.6(1.9-142.0)$ & $0.5(0.1-1.5)$ \\
\hline $\begin{array}{l}\text { Healthy controls ( } \mathrm{n}=8 \text {, undiluted } \\
\text { fluid) }\end{array}$ & $33.4(3.5-89.3)$ & $51.2(11.6-148.8)$ & $0.4(0.1-1.2)$ \\
\hline
\end{tabular}




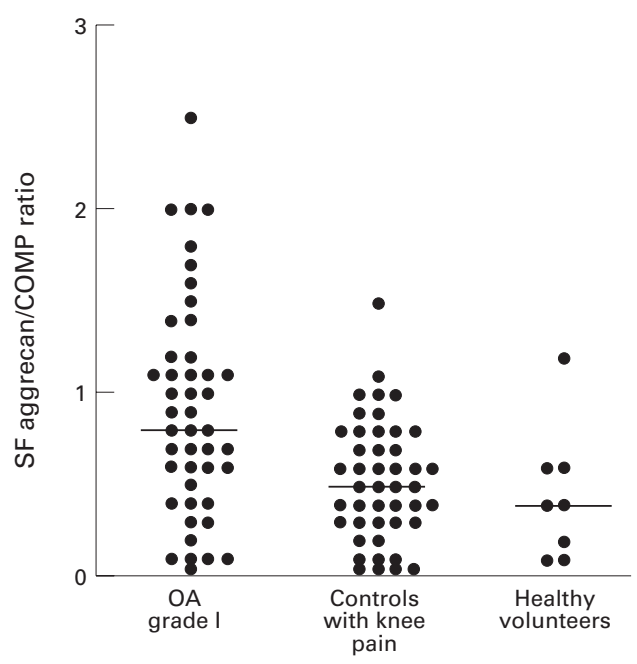

Ratios between aggrecan and COMP fragments-

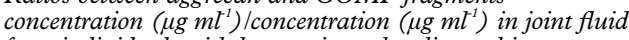
from individuals with knee pain and radiographic osteoarthritis grade I, controls with knee pain and normal radiographs, and healthy controls. For explanation, see text.

\section{Discussion}

The possibilities of diagnosing early osteoarthritis are limited at present. This precludes studies of the phases of the disease which are essential for the initiation and perpetuation of the pathophysiological process.

Joint space narrowing in the tibiofemoral joint observed on weight bearing plain radiographs is a fairly specific method. It is, however, far too insensitive for the detection of early changes. The lack of tools for early diagnosis hampers efforts to design means of monitoring the efficacy of potentially disease modifying agents. Such agents, provided they can be given early in the disease before irreversible changes are seen in the form of loss of cartilage, should be capable of retarding disease progression. ${ }^{14}$ The pathological process in the cartilage most probably starts much earlier than the appearance of radiographic changes and probably also before changes can be detected by magnetic resonance imaging ${ }^{15}$ or arthroscopic inspection. ${ }^{4}$ Therefore new instruments for early diagnosis are needed. Quantification of cartilage macromolecules or fragments thereof released into the synovial fluid in joint damaging processes has received attention as one such instrument. Indeed, studies supporting the feasibility of this approach have recently been reported (for review see ${ }^{3}$ ). In the present study we show that the relative content in synovial lavage fluid of two cartilage markers differs between patients with knee pain and radiographic changes compatible with osteoarthritis and patients with knee pain without radiographic changes. The relative content of aggrecan antigens in the fluid was higher in patients with radiographic osteoarthritis, which suggests increased turnover in the cartilage of this macromolecule. The differences between the two groups were thus significant despite the fact that some individuals in the radiographically normal group obviously might have a milder preradiographic stage of osteoarthritis. This might also in part explain the marked overlap of the ratios between the groups. We chose to compare the ratios of aggrecan and COMP since in traumatic and arthritic conditions, for example, rheumatoid arthritis and reactive arthritis, increased release of aggrecan has been found early in the disease course. ${ }^{578}$ In contrast, although increased COMP release is observed in these conditions, the increase is less pronounced and-at least in rheumatoid arthritis - follows a somewhat different time course ( ${ }^{9}$; Saxne T, unpublished). The results from the present study suggest that the turnover of aggrecan is also markedly increased in the osteoarthritis process and that the turnover of COMP is affected differently.

The finding of low aggrecan/COMP ratios in the healthy knee control group are in support of a model where increased release of aggrecan relative to COMP indicates a pathological shift in the cartilage turnover.

Furthermore, this study shows similar trends to those found in previous observations of the relative content of these cartilage macromolecules in synovial fluid from healthy individuals as well as from patients with advanced osteoarthritis. Thus in synovial fluids obtained from healthy athletes, ratios between aggrecan and COMP concentrations of mean 0.8 (range 0.4 to 1.3 ) were found. ${ }^{10}$ In patients with advanced osteoarthritis, as determined radiographically, the ratios vary between 0.3 and 2.8 with a mean ratio of 1.3 and a distribution of individual values closely resembling those obtained in the present study. ${ }^{9}$ In a small group of hospital recruited patients with minor arthroscopic changes compatible with osteoarthritis, we have previously found aggrecan/ COMP ratios in the same range as in the present study. ${ }^{10}$ The analogy between other observations and those of the present study suggests that the use of knee joint lavage for retrieval of fluid for analysis of cartilage fragments in the knee joint is a method that can be applied in situations where it is difficult to obtain synovial fluid. Mixing of injected fluid with the small residual volume of synovial fluid is a critical point. However, the fact that we obtained similar results in studies where synovial fluid was obtained without the lavage procedure suggests that the form of mixing procedure used is adequate. Furthermore, the dilution reproducibility has been shown to be satisfactory in the original study of the lavage method. ${ }^{13}$ A direct comparison of the concentrations of the markers between the groups was not possible since the degree of dilution after lavage obviously cannot be measured.

Our study shows that it is possible to obtain joint fluid samples by a lavage procedure in knee joints containing minimal amounts of synovial fluid. Furthermore, we believe that the quantification of cartilage macromolecules in such fluids and the use of concentration ratios should be useful for assessing cartilage involvement in osteoarthritis. The ratio is a ratio of concentrations and simply aims at defining a way to overcome the difficulties of comparing concentrations when retrieving synovial fluid 
by a lavage procedure. This study clearly shows the feasibility of this approach, which opens new possibilities also for the study of early, preradiographic, stages of osteoarthritis. However, it should be emphasised that the groups in the present study were primarily defined by the presence or absence of radiological features of osteoarthritis. Thus the diagnostic potential of these measurements in early osteoarthritis has yet to be determined. The diagnostic as well as the prognostic value of quantification of aggrecan and COMP in early osteoarthritis can only be evaluated in long term follow up of cohorts such as the one described in this study.

We thank the staff of Spenshult's Hospital for Rheumatic Diseases for good clinical and secretarial assistance, Mr Gunnar Severinsson, Carmona Business Concept, Halmstad, and $\mathrm{Mr}$ Severinsson, Carmona Business Concept, Halmstad, and $\mathrm{Mr}$
Jonas Winge, Lund, for excellent assistance in computer work, Jonas Winge, Lund, for excellent assistance in computer work,
and Mrs Mette Lindell for skilful technical assistance. Grants and Mrs Mette Lindell for skilful technical assistance. Grants ish Medical Research Council, Konung Gustaf V:s 80-årsfond, Medicinska fakulteten i Lund, Hallands Läns Landsting, Medicinska fakulteten i Lund, Hallands Läns
Crafoords, Kocks, Osterlunds, and Svartz Stiftelser.

1 Altman RD. Overview of osteoarthritis. Am J Med 1987;83(suppl 4B):65-9.

2 Badley E, Tennant A Impact of disablement due to rheumatic disorders in a British population: estimates from the Calderdale Rheumatic Disablement Survey. Ann Rheum Dis 1993;52:6-13.
3 Poole AR. Immunochemical markers of joint inflammation, skeletal damage and repair: where are we now? Ann skeletal damage and rep
Rheum Dis $1994 ; 53: 3-5$.

4 Lysholm J, Hamberg P, Gillquist J. The correlation between osteoarthrosis as seen on radiographs and on arthroscopy. Arthroscopy 1987;3:161-5.

5 Saxne T, Heinegård D. Matrix proteins: potentials as body fluid markers of changes in the metabolism of cartilage and bone in arthritis. J Rheumatol 1995;22(suppl 43); $71-4$.

6 Messner K, Gillquist J, Björnsson, S, Lohmander LS. Proteoglycan fragments in rabbit joint fluid correlated to arthrosis stage. Acta Orthop Scand 1993;64:312-6

7 Saxne T, Heinegård D. Synovial fluid analysis of two groups of proteoglycan epitopes distinguishes early and late cartilage lesions. Arthritis Rheum 1992;35:385-90.

8 Lohmander LS, Dahlberg L, Ryd L, Heinegård D. Increased levels of proteoglycan fragments in knee joint Increased levels of proteoglycan fragments in knee join
fluid after injury. Arthritis Rheum 1989;32:1434-42.

9 Saxne T, Heinegård D. Cartilage oligomeric matrix protein a novel marker of cartilage turnover detectable in synovial a novel marker of cartilage turnover detectable in
fluid and blood. Br J Rheumatol 1992;31:583-91.

10 Lohmander LS, Saxne T, Heinegård D. Release of cartilage oligomeric matrix protein (COMP) into joint fluid after knee injury and in osteoarthritis. Ann Rheum Dis 1994;53:8-13.

11 Ahlbäck S. Osteoarthrosis of the knee: a radiographic investigation. Acta Radiol (Stockholm) 1968;suppl 277:7-72. 12 Kellgren JH, Jeffrey M, Ball J. Atlas of standard radiographs
(The epidemiology of chronic rheumatism; vol 2). Oxford: Blackwell Scientific, 1963

13 Geborek P, Saxne T, Heinegård D, Wollheim FA. Measurement of synovial fluid volume using albumin dilution upon intraarticular saline injection. J Rheumatol 1988;15:57intraart 60 .

14 Wollheim FA. New insight into joint damage from analysis of released biochemical markers. Br J Rheumatol 1994; of releasec $1-4$.

15 Fernandez-Madrid F, Karvonen RL, Teitge RA, Miller PR, Negendank WG. MR features of osteoarthritis of the knee. Magn Reson Imaging 1994;12:703-9. 